Les vagues observées dans le sillage des bateaux ont été étudiées depuis longtemps, et l'on croyait le phénomène bien compris.

Mais l'étude des bateaux rapides a révélé un sillage plus étroit que prévu par la théorie classique de Kelvin. Ce qui, finalement, peut s'expliquer assez simplement...

\title{
Du neuf dans les sillages
}

Marc Rabaud (marc.rabaud@u-psud.fr) et Frédéric Moisy Laboratoire FAST, CNRS UMR 7608, Université Paris-Sud, 91405 Orsay

Chacun de nous a déjà vu les petites vagues formant un « V » qui accompagnent un canard nageant sur un étang (fig. 1). Un sillage similaire est visible à l'arrière d'un bateau avançant sur eau plate, et plus généralement derrière tout obstacle se déplaçant pas trop lentement à la surface d'un liquide. Au-delà de son aspect esthétique, l'existence de ce sillage a de nombreuses conséquences car les vagues qui le composent transportent de l'énergie loin du bateau. On peut s'en apercevoir à la manière dont un bateau rentrant un peu trop vite dans un port secoue les autres bateaux amarrés. Cette énergie rayonnée correspond au travail d'une force qui freine le bateau, force dite de "traînée de vague ". À haute vitesse, cette force de traînée de vague devient même la contribution dominante à la résistance à l'avancement des navires.

Le premier scientifique à s'être intéressé à cet effet est sans doute William Froude qui, au XIX ${ }^{\mathrm{e}}$ siècle, initia l'utilisation de maquettes pour des essais de traction en hydrodynamique navale [1]. C'est toutefois le nom de Kelvin qui est associé aux sillages de vagues car c'est lui qui, en 1887 (il portait alors encore le nom de William Thomson), fut le premier à expliquer la forme du sillage [2]. En développant pour cette occasion la méthode dite de la phase stationnaire, qui s'est avérée depuis très riche dans de nombreuses branches de la physique, il démontra que la crête des ondes en eaux profondes dessine des formes " en trompette", dont les points de rebroussement sont situés sur les bords du «V» (fig. 2). Le résultat le plus surprenant est que le demi-angle au sommet de ce " $\mathrm{V}$ " vaut $\alpha_{0}=\arcsin (1 / 3)=19,47^{\circ}$, et ceci quelles que soient la vitesse $U$ et la taille $L$ de l'obstacle en mouvement. Ce très beau résultat, essentiellement géométrique, est devenu un grand classique de la physique des vagues, et il est souvent présenté en cours ou dans les livres d'enseignement pour illustrer les propriétés de dispersion des ondes de gravité (voir encadré 1).
SHIP WAVES.

Wave Pattern.

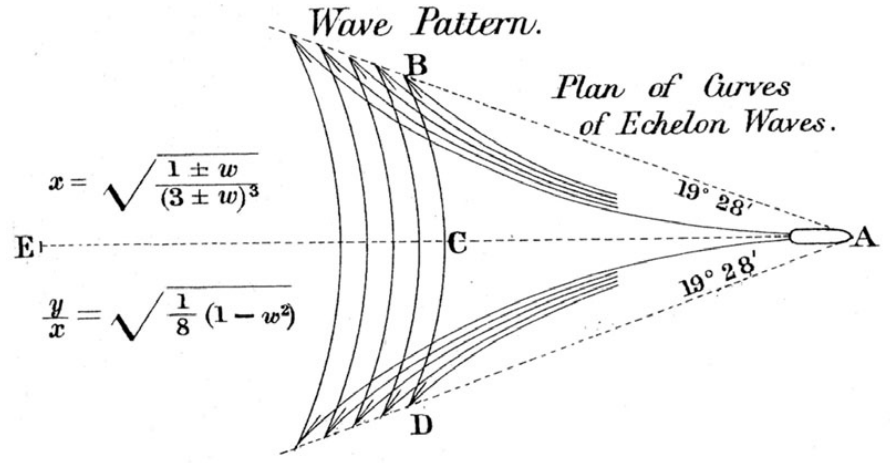

2. Illustration issue de l'article de Lord Kelvin montrant les lignes isophases des vagues d'accompagnement " en chevrons " d'un navire se déplaçant selon ECA [2]. On y distingue les ondes transverses $B C D$ qui se propagent dans la direction du déplacement du bateau, et les ondes divergentes BA et DA qui s'éloignent de la trajectoire du bateau. Les points de rebroussement B et D constituent les bords du sillage en « $V$ », dit de Kelvin. Les équations reportées sur la figure donnent une représentation paramétrique (y, $\mathrm{x}$ ) des lignes isophases, où le paramètre w varie dans l'intervalle [-1, 1]. 


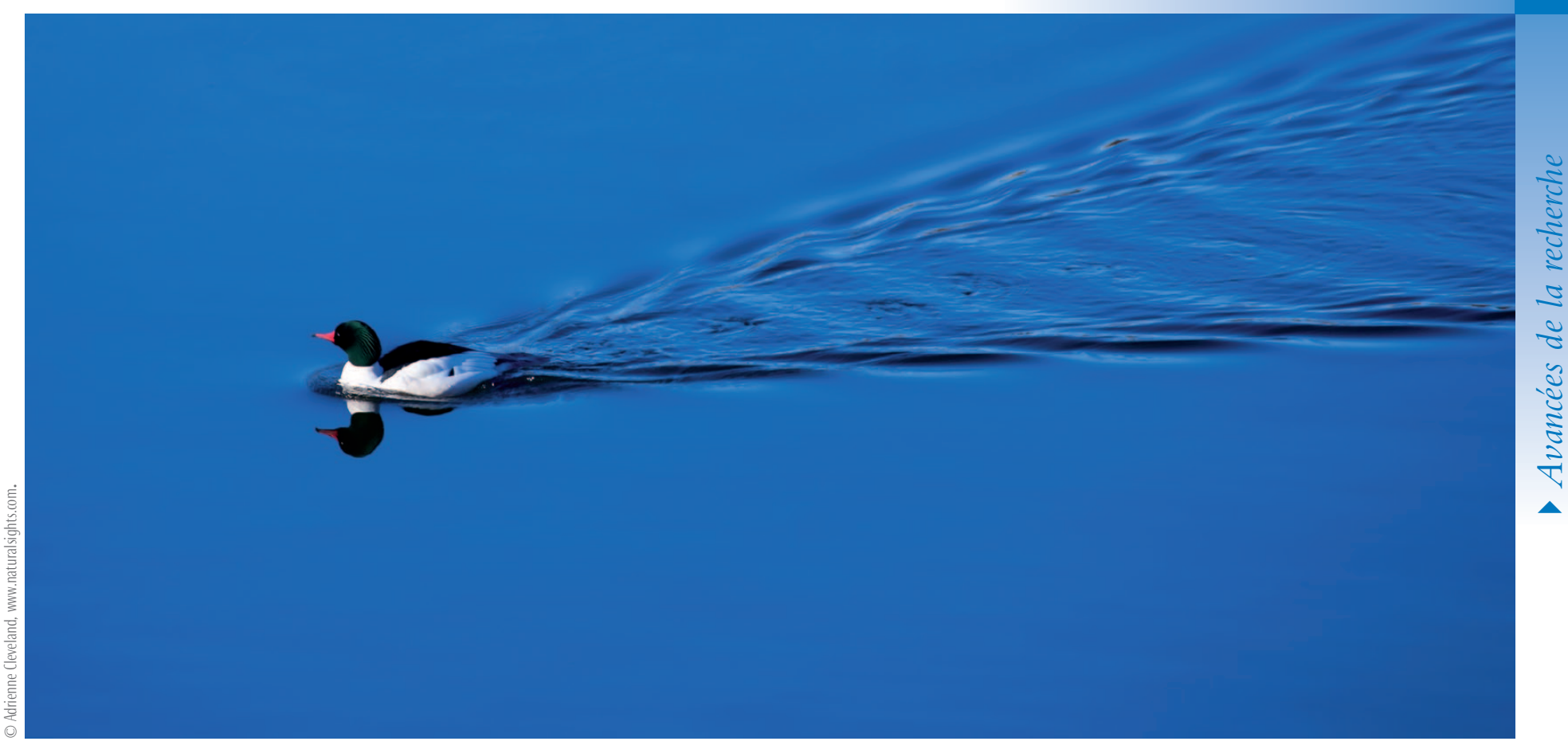

1. Canard nageant à la surface d'un lac.

\section{La théorie de Kelvin apparemment mise en défaut}

Pourtant, en réalisant une démonstration de cours avec des étudiants autour d'une piscine, nous avons constaté que les mesures n'étaient clairement pas en accord avec la belle théorie de Kelvin. Plus la vitesse de l'obstacle était grande et plus l'angle du sillage diminuait ! Ce résultat semblait d'ailleurs confirmé par certaines photos de vedettes rapides montrant des sillages étroits.

Nous avons donc voulu mieux analyser ce phénomène. Il y a toutefois deux difficultés à surmonter d'un point de vue expérimental : (i) si l'on travaille en piscine avec des maquettes forcément relativement petites, il y a des effets correctifs dus aux forces capillaires, ce qui vient compliquer la géométrie du sillage [9], et (ii) si l'on travaille à l'échelle 1:1 à partir de photographies de sillages de vrais bateaux, il est souvent difficile de mesurer précisément les angles à cause d'effets de perspective. Nous avons contourné ces difficultés en travaillant à partir d'images aériennes, et donc en incidence presque normale, disponibles sur Internet grâce à Google Earth $^{(}{ }^{\complement}$. En sélectionnant des images audessus de lacs ou du littoral pour avoir une bonne résolution, nous avons mesuré des angles de sillages qui varient entre 7 et $20^{\circ}$ (fig. 3 et réf. [3]).

Autre avantage de ces images aériennes, elles sont disponibles avec une calibration spatiale qui permet de mesurer la longueur $L$ des bateaux d'une part, mais aussi de calculer

\section{La relation de dispersion des ondes de gravité} en eau profonde

Contrairement aux ondes sonores qui voyagent toutes à la même vitesse, les ondes à la surface d'un liquide ont une vitesse qui dépend de leur longueur d'onde, du moins lorsque celle-ci est très inférieure à la profondeur d'eau. On dit qu'elles sont "dispersives », car un paquet d'onde contenant plusieurs longueurs d'ondes va se disperser lors de sa propagation. Pour des ondes de grande longueur d'onde où seule la force de gravité intervient, on peut montrer que la relation qui relie la pulsation $\omega$ des ondes à leur nombre d'onde $k$ s'écrit : $\omega^{2}=g k$, où $g$ est l'accélération de la pesanteur.

Cette relation n'étant pas linéaire, la vitesse de groupe de chaque onde (vitesse de propagation de l'énergie), $c_{g}(k)=d \omega / d k$, vaut la moitié de sa vitesse de phase, $c_{\varphi}=\omega / k$ (vitesse $d^{\prime}$ avancée des crêtes ou des creux de l'onde). Ce phénomène est bien visible lorsque l'on lance un caillou dans l'eau : il apparaît un paquet d'onde circulaire, qui se propage à une vitesse de groupe liée à la taille du caillou. Ce paquet d'onde est constitué d'ondes qui semblent naître à l'arrière et viennent mourir à l'avant du paquet.

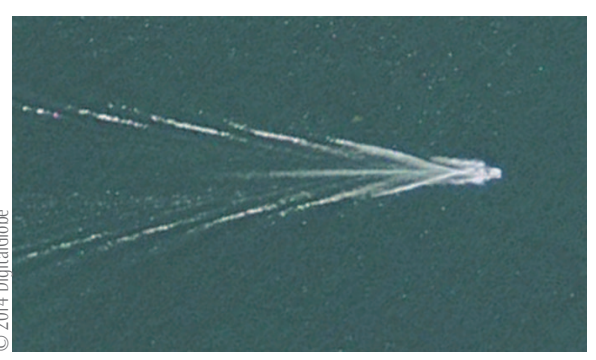

3. Vue aérienne d'un sillage étroit, d'environ 9 degrés, d'une vedette rapide sur le lac Ontario près de Toronto.

leur vitesse $U$. En effet, à partir de la mesure de la longueur d'onde visible dans le sillage et de la direction de propagation de ces ondes, la condition de stationnarité (présentée dans l'encadré 2) permet de déterminer la vitesse du bateau. Nous avons alors tracé la valeur des angles de sillages en fonction du nombre de Froude de coque, nombre sans dimension défini par $\mathrm{Fr}=U /(g L)^{1 / 2}$, et qui compare la vitesse du bateau à la vitesse des ondes de gravité ayant pour longueur d'onde la longueur du bateau. La figure 4 montre que les résultats se rassemblent assez bien sur une courbe unique. Tant que Fr $<0,5$, les mesures sont en accord avec la prédiction de Kelvin d'un angle constant. Mais au-delà de cette valeur elles montrent une nette décroissance, suivant une loi en $1 / \mathrm{Fr}$. Ces observations ont été confirmées en simulant numériquement l'émission d'ondes de gravité par un objet en mouvement [4], et ont été récemment confirmées analytiquement par des collègues de l'ESPCI [5]. 


\section{Analyse classique du sillage en " $\mathrm{V}$ »} encadré 2

L'analyse de Kelvin est basée sur le modèle simplifié d'une perturbation ponctuelle, censée exciter tous les nombres d'ondes (bruit blanc). Après un transitoire possible au démarrage du bateau, seules seront observées les ondes stationnaires dans le référentiel du bateau.

Considérons une onde de nombre d'onde $k$, se propageant dans la direction $\theta$ (figure E1 ci-dessous). Les crêtes de cette onde voyagent par rapport à l'eau à la vitesse de phase $c_{\varphi}$. Pour que les crêtes restent immobiles dans le référentiel du bateau, il faut que la vitesse de l'eau le long de la direction de propagation de l'onde s'oppose exactement à la vitesse de phase, ce qui donne la condition de stationnarité : $\mathrm{c}_{\varphi}=\omega / k=U \cos \theta(k) \quad(1)$.

La figure E1 nous montre que si le milieu était non dispersif, le sillage construit avec toutes les positions successives $\mathrm{M}$ serait localisé sur la droite OI (c'est le cas pour l'onde de choc d'un avion supersonique). Mais pour les ondes de gravité, comme l'énergie se propage à la vitesse de groupe $C_{g}$ qui est égale à la moitié de la vitesse de phase $C_{\varphi}$, elle ne parvient qu'au point $\mathrm{H}$, milieu de $\mathrm{MI}$, au moment où le bateau arrive en $\mathrm{O}$. L'énergie n'est donc pas sur la droite $\mathrm{Ol}$, mais sur la droite $\mathrm{OH}$, qui fait un angle $\alpha(\mathbf{k})$ spécifique pour chaque vecteur d'onde [6].

Pour chacun des nombres d'onde $k$, la condition de stationnarité impose la direction de propagation $\theta(k)$. II existe donc un angle de sillage $\alpha(k)$ qui dépend de $k$ et que l'on peut déterminer géométriquement (fig. E1). Par exemple, lorsque $\theta$ est nul, $\alpha$ l'est aussi, ce qui correspond aux plus petits $k$ admis par la relation (1) : il s'agit des ondes transverses, de nombre d'onde $k_{g}=g / U^{2}$, dont les crêtes sont perpendiculaires à l'avancée du bateau. Mais $\alpha$ est aussi nul lorsque $\theta=90^{\circ}$ (ondes de très petite longueur $d^{\prime}$ onde). Entre ces deux extrêmes, $\alpha$ présente un maximum égal à $\alpha_{0}=\arcsin (1 / 3) \approx 19,47^{\circ}$, pour un nombre d'onde intermédiaire égal à $k_{0}=3 k_{g} / 2$ : aucune onde accompagnant le bateau ne peut être observée au-delà de cet angle. Mais le maximum d'énergie, lui, n'est pas nécessairement à cet angle : tout dépend de la façon dont les différents nombres d'onde vont être effectivement alimentés en énergie par la perturbation.

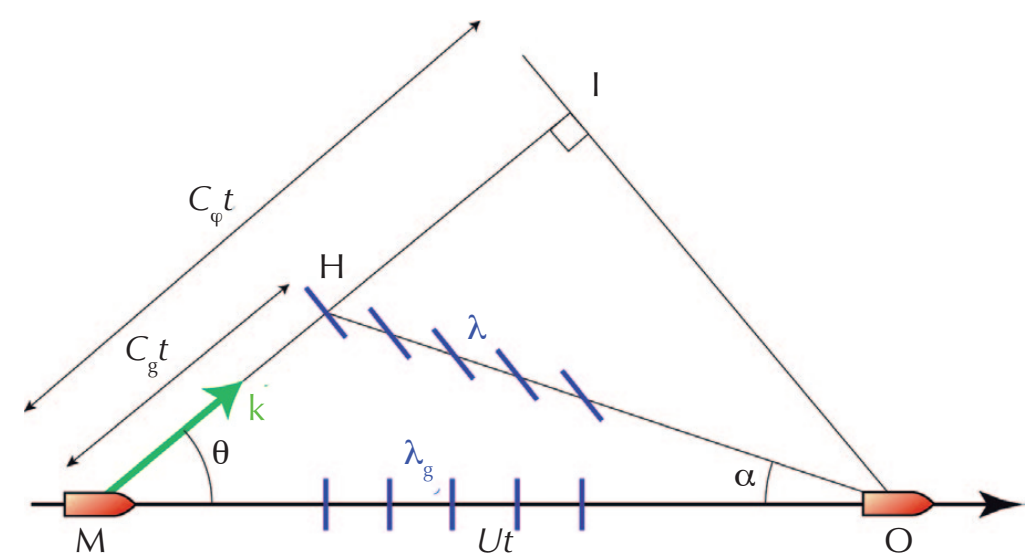

E1. Construction géométrique pour une onde de vecteur d’onde $\mathbf{k}$ et émise lorsque le bateau était en M dans la direction définie par $\theta$. Pour être stationnaires vis-à-vis du bateau en 0 , les ondes qui ont été émises dans la direction $\theta$ doivent avoir leur crête parallèle à 01 .

\section{Références}

1• 0 . Darrigol, Worlds of Flow: A History of Hydrodynamics from the Bernouillis to Prandtl, Oxford University Press (2005).

2•W. Thomson, "On ship waves", Lecture delivered at the "Conversazione" in the Science and Art Museum, Edinburgh, on 3 Aug. 1887, Institution of Mechanical Engineers, Minutes of proceedings (1887) 409-434.

3- Liste des images disponible à l'adresse : www.fast. u-psud.fr/ $\sim$ moisy/papers/2013_rabaud_prl_sm.pdf

4• M. Rabaud et F. Moisy, "Ship wakes: Kelvin or Mach angle?", Phys. Rev. Lett. 110 (2013) 214503.

\section{\>}

\section{Sillage de Kelvin ou cône de Mach ?}

Un nombre de Froude de transition de l'ordre de 0,5 correspond aux vitesses pour lesquelles les ondes émises ont des longueurs d'onde qui deviennent comparables à la longueur de la coque. C'est aussi à partir de ces vitesses que les bateaux rapides dépassent leur "vitesse limite de coque " et sortent partiellement de l'eau : on dit qu'ils " déjaugent " ou qu'ils " partent au planning ». Il est intéressant de noter que la décroissance en $1 / \mathrm{Fr}$ de l'angle du sillage observée pour $\mathrm{Fr}>0,5$ est analogue à celle en $1 /$ Ma pour l'angle du cône de Mach des avions supersoniques, où le nombre de Mach $\mathrm{Ma}=U / c$ compare la vitesse de l'avion à la vitesse du son. Ce résultat suggère que, dans le problème du bateau, la vitesse des ondes de gravité dont la longueur d'onde est $L$ joue un rôle un peu équivalent à une "vitesse du son ». Ce qui est surprenant ici, c'est que c'est un modèle non dispersif (vitesse des ondes indépendante de leur longueur d'onde) qui se trouve le mieux décrire le sillage des bateaux à haute vitesse en milieu dispersif. Pourquoi?

Pour répondre à ce paradoxe, il faut repartir de l'explication classique du sillage de Kelvin (détaillée dans l'encadré 2), qui montre qu'à chaque nombre d'onde $k$ on peut associer un angle $\alpha(k)$ selon lequel l'énergie de l'onde sera rayonnée, et qui vaut au maximum l'angle de la théorie de Kelvin, soit $19,47^{\circ}$. Si tous les nombres d'onde sont bien alimentés en énergie par le bateau, alors l'angle de Kelvin sera visible. Mais dans la réalité, un bateau ne va pas nécessairement exciter tout le spectre des nombres d'onde : le spectre d'énergie effectivement rayonné dépendra a priori de la forme exacte de la coque, de sa position dans l'eau, et de la nature de l'écoulement autour d'elle (turbulence, présence de déferlement, etc.). Sans entrer dans toutes les subtilités de l'architecture navale, nous pouvons nous contenter de considérer la plus grande échelle pertinente de ce problème qui est la longueur de la coque du bateau. Nous considérons donc une simple surpression localisée et axisymétrique, de taille $L$, se déplaçant à la surface de l'eau [7]. Une telle surpression induit une déformation de la surface de l'eau qui simule la présence d'un obstacle rigide. On peut montrer qu'une telle perturbation génère des vagues d'amplitude maximum pour un nombre d'onde de 
l'ordre de $1 / \mathrm{L}$. Si ce nombre d'onde est voisin de $k_{g}=g / U^{2}$, alors l'angle de Kelvin sera bien alimenté en énergie, et le sillage aura un maximum d'amplitude à l'angle classique $\alpha_{0} \approx 19,5^{\circ}$. Mais, à plus haute vitesse, les longueurs d'onde nécessaires à la construction du sillage classique, d'ordre $k_{g}{ }^{-1}=U^{2} / g$, deviennent très supérieures à la taille $L$, et ne pourront donc plus être alimentées efficacement par le bateau. Seules les plus petites longueurs d'ondes, de l'ordre de $L$, vont donc être visibles, et cela dans une direction correspondant à un angle $\alpha(k=1 / L)$ inférieur à l'angle de Kelvin, ce qui explique l'observation de sillages plus étroits à grandes vitesses. Plus quantitativement, on peut montrer que l'on a $\alpha(k) \approx\left(k_{0} / k\right)^{1 / 2}$ à grand $k$, ce qui donne un maximum d'énergie rayonnée selon l'angle $\alpha \approx\left(k_{0} L\right)^{1 / 2} \sim(g L)^{1 / 2} / U:$ on retrouve bien une loi de type cône de Mach en $\alpha \cong 1 / \mathrm{Fr}$ (fig. 4), comme observée dans les images aériennes, ainsi que dans nos simulations numériques (fig. 5). Physiquement, tout se passe comme si la taille du bateau sélectionnait une "vitesse du son » effective, donnée par la vitesse de groupe des ondes dont la longueur d'onde coïncide avec la taille du bateau. Finalement, l'analyse proposée par Kelvin, qui stipule qu'il n'existe aucune onde stationnaire au-delà d'un angle de $19,5^{\circ}$, reste bien vérifiée. Mais si l'on veut décrire l'angle du maximum d'amplitude des ondes, qui est celui que l'on observe en pratique, il est nécessaire de prendre en compte explicitement la taille finie du bateau.

Richard Feynman, dans son célèbre cours de physique [8], disait des ondes de surface: "they are the worst possible example, because they are in no respects like sound and light; they have all the complications that waves can have." Et pourtant, malgré cette complexité liée à la nature dispersive des ondes, c'est finalement ici une physique très simple qui se trouve décrire le mieux l'angle de sillage des bateaux rapides : celle du cône de Mach des ondes non dispersives! Il vous suffit d'ailleurs d'essayer, en déplaçant votre doigt ou une aiguille à la surface de l'eau dans votre baignoire. Au-delà de $50 \mathrm{~cm} / \mathrm{s}$ environ, vous observerez un sillage d'autant plus étroit que la vitesse est élevée, même si ici les effets capillaires sont importants - mais ceci est une autre histoire [9]...

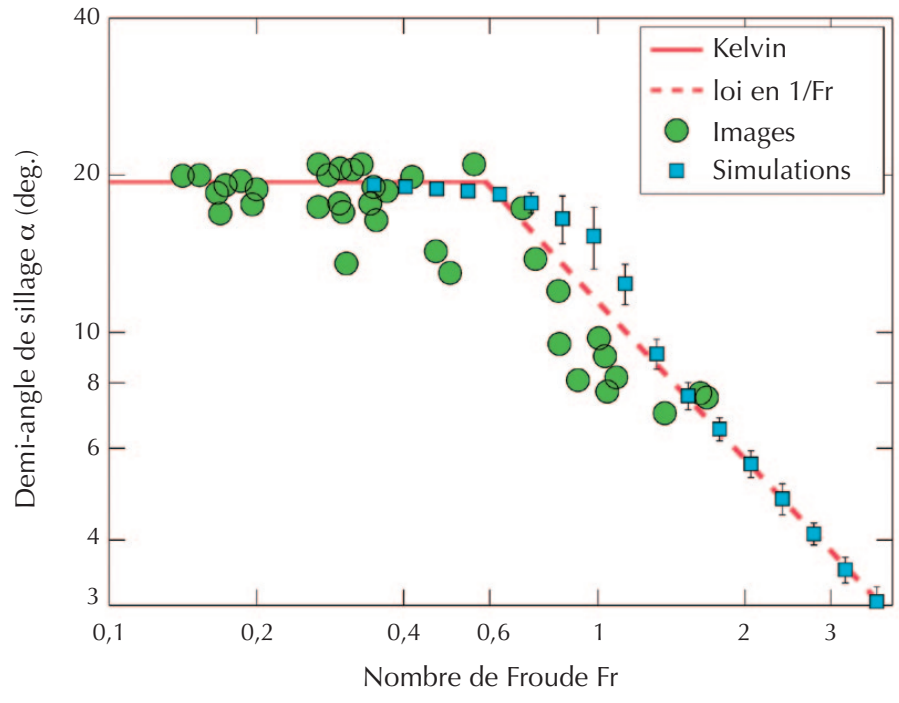

4. Évolution du demi-angle $\alpha$ du sillage observé en fonction du nombre de Froude $\mathrm{Fr}=\mathrm{U} /\left(\mathrm{gL} \mathrm{L}^{1 / 2}\right.$, montrant une transition entre le régime de Kelvin (angle constant égal à 19,47) et le régime en 1/Fr à haute vitesse.

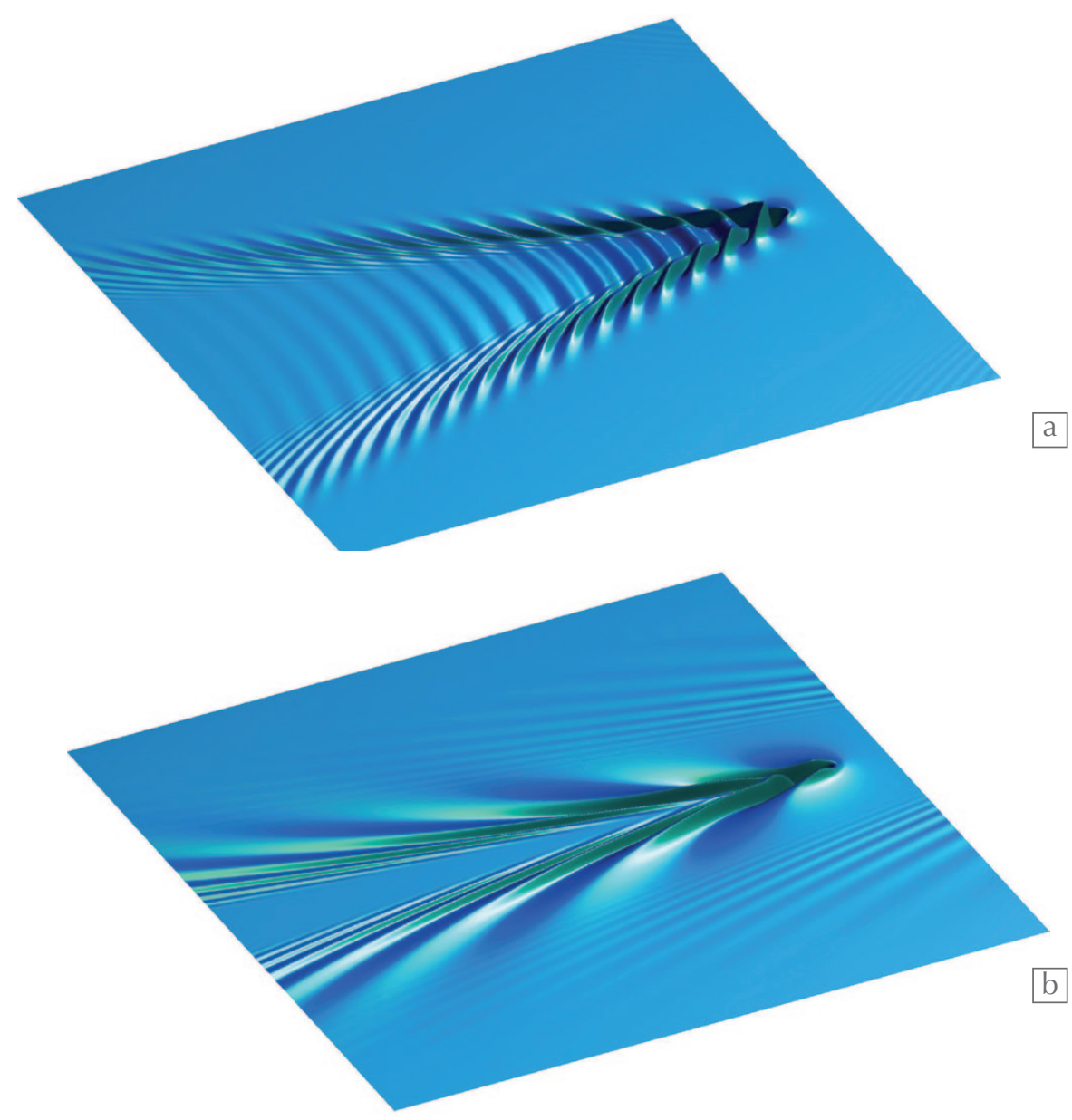

5. Simulation numérique d'un sillage induit par une perturbation de pression gaussienne se déplaçant à nombre de Froude $\mathrm{Fr}=0,5$ (a) et 1,5 (b). 